\title{
Hábitos cognitivos, ejecutivos, afectivos y éticos modificados durante la cuarentena en Ecuador
}

\section{Resumen}

Globalmente la aparición del Covid-19 ha generado un estado preocupante. El presente estudio se enfoca en Ecuador, siendo uno de los países latinoamericanos más afectados, tanto por el número de mortandad, como de contagios. Es en este sentido que la investigación pretende aportar con información sobre hábitos cognitivos, ejecutivos, afectivos y éticos durante situaciones de crisis. Se realizó una exploración cuantitativa de alcance descriptivo, con el objetivo de conocer la percepción de modificación en los tipos de hábitos desde la perspectiva de la psicología cognitiva y del aprendizaje. La muestra estuvo constituida por 173 personas con un rango de edad de 24 a 60 años que residen en Ecuador. Para ello se aplicó una encuesta con preguntas semi-estructuradas, los resultados evidenciaron una modificación en ciertos hábitos cognitivos y afectivos. No obstante, los hábitos ejecutivos y éticos han quedado intactos, sin modificación alguna. Se concluyó que en la modificación de hábitos cognitivos prevalece la percepción de preocupación por el desastre y falta de atención. Asimismo, en los hábitos afectivos se evidenció una modificación relacionada a sentimientos de tristeza, ansiedad, sensibilidad al cambio y miedo dominantes durante el confinamiento. 


\begin{abstract}
Globally, the appearance of Covid-19 has generated a worrying situation. The present study focuses on Ecuador, being one of the most affected Latin American countries, both in terms of the number of deaths and contagions. In this sense, the research aims to provide information on cognitive, executive, affective and ethical habits during crisis situations. A quantitative exploration of descriptive scope was carried out, with the objective of knowing the perception of modification in the types of habits from the perspective of cognitive psychology and learning. The sample consisted of 173 people between 24 and 60 years of age living in Ecuador. A survey with semi-structured questions was applied, the results showed a modification in certain cognitive and affective habits. However, executive and ethical habits have remained intact, without any modification. It was concluded that in the modification of cognitive habits, the perception of preoccupation with disaster and lack of attention prevailed. Likewise, in affective habits, there was evidence of a modification related to feelings of sadness, anxiety, sensitivity to change and dominant fear during confinement.
\end{abstract}

Keywords: habits, quarantine, Covid-19. 


\section{Introducción}

La Organización Mundial de la Salud (2020) ha realizado varios registros del Covid-19, que hasta marzo 27 del 2020 sobrepasó los 500.000 casos. Varios países han implementado medidas de salud ante el estado de emergencia internacional que han impactado en varios sectores, tales como aviación, límites, transporte marítimo, sectores turísticos, sectores de salud, sector económico y otros. Por ello, el Covid-19 es declarado como emergencia de salud en enero del 2020, al ser un virus de alta proliferación, convirtiéndose en pandemia en marzo de ese mismo año.

La situación del Ecuador, según El Comercio (2020), indicó que hasta el 16 de abril de 2020 el país constató 8225 enfermos y 1035 personas que fallecieron en el contexto de coronavirus. Este fenómeno mundial ha afectado aproximadamente a 2,4 millones de personas contagiadas y 171.000 muertos registrados (RTVE, 2020). Anteriormente a la existencia del coronavirus, se habían realizado estudios de hábitos relacionados con enfermedades crónicas, tales como obesidad, diabetes, enfermedades cardiovasculares e hipertensión, que se encuentran directa o indirectamente relacionadas con hábitos inadecuados de alimentación y sedentarismo (MacMillan, 2007). La interrogante primordial del estudio es ¿Qué hábitos se modifican durante la situación de crisis por la cuarentena por Covid-19? El estado de crisis es un momento de vulnerabilidad que se origina a partir de un suceso precipitante e inesperado. Ello conlleva varios síntomas, tales como: emociones negativas (enfado, ansiedad, estrés y tristeza), deficiente interacción social, inferencias negativas, falta de concentración en la actividad académica, pérdida de interés en actividades placenteras, falta de apetito, alteración en el sueño y desorganización en la rutina diaria (Echeburúa \& Corra, 2007; Estrada et al., 2017).

Para entender el tema, el actual estudio se inclina al marco de la teoría cognitiva y aprendizaje, siguiendo el lineamiento de Marina (2012), los hábitos son esquemas mentales dinámicos que se encargan de recoger, guardar y procesar información. De tal modo que nos permiten percibir el mundo y accionar en él. Los hábitos se adquieren mediante la repetición de conductas y persisten facilitando operaciones motoras, afectivas y cognitivas. Ello implica que pueden ser de varios tipos: cognitivos, afectivos, ejecutivos y éticos. No obstante, según Estrada et al. (2017), en estados de crisis los hábitos se ven modificados.

En este sentido, Marina (2012) sostiene que los hábitos cognitivos se refieren a la capacidad de atender, concentrarse, recordar, tomar decisiones, pensar de manera organizada y orientarse de manera espacial. Estos hábitos están dirigidos por juicios razonables. A su vez, en estados de crisis, Estrada et al. (2017) indicaron que se observan modificaciones a nivel cognitivo: confusión y desorientación, dificultades en concentración, memoria y atención, dificultad en toma de decisiones, pensamientos desorganizados, preocupación del desastres y detalles insignificantes.

Otro tipo de hábitos son los afectivos, vinculados a la identificación, autocontrol emocional y valoración de emociones, enfatizando en estados positivos afectivos. A su vez, estos hábitos involucran relaciones sociales saludables (Marina, 2012). Cuando aparecen sucesos precipitantes o de crisis surgen las siguientes emociones negativas: culpabilidad, ansiedad, cansancio, 
hipersensibilidad a estímulos sensoriales, tristeza e ira. En el ámbito de habilidades sociales puede crear dependencia, conflictos familiares y aislamiento (Estrada et al., 2017).

Por otra parte, los hábitos ejecutivos se refieren a organización de horarios, hábitos de estudios, alimentación y control conductual (Marina, 2012). Al ocurrir una situación precipitante, los hábitos ejecutivos cambian, existe falta de control conductual, lo que incrementa conductas agresivas e impulsivas, desorganización en horarios y en ejecución en actividades variadas tales como estudio, horas de dormir, alimentación y ejercicios físico. De esta manera, existiría un bloqueo en el pensamiento que produce hábitos sedentarios o pasivos, lo que significa que la persona se aísla y puede percibir que sus horarios de dormir, alimentarse y estudiar no tienen una organización. Asimismo, puede realizar las actividades anteriormente mencionadas con exceso, sin regulación (Estrada et al., 2017).

Dentro de los hábitos éticos, Marina (2012) refiere a la capacidad de actuar siguiendo valores éticos, religiosos o espirituales. En crisis, Estrada et al. (2017) evidenciaron dudas en cuanto a pensamientos de carácter religioso y espiritual, igualmente, en ciertos casos podría incrementarse la ayuda solidaria por parte de la gente hacia personas que necesitan o también puede evidenciarse una falta de solidaridad (Estrada et al., 2017; Ayuntamiento de Vitoria Gasteiz, 2020).

La investigación que se realiza tiene amplios marcos teóricos (Caballero \& Campos, 2018; Cabrera, 2006; Leija et al., 2011; MacMillan, 2007; Solano et al., 2017; Trujillo, 2018) acerca del tema de hábitos saludables y sedentarios, asimismo, en cuanto a tipo de hábitos como los cognitivos, afectivos, ejecutivos y éticos.

Respecto del tema de estudio, debe aclararse que las dimensiones articuladas a la problemática de la modificación de hábitos cognitivos, ejecutivos, afectivos y éticos en cuarentena Covid19 han sido estudiadas de diversas formas, desde el enfoque cognitivo y aprendizaje. En la actualidad, la situación del Covid-19 ha afectado mundialmente, por ello, estudiar los hábitos durante cuarentena, con personas que residen en Ecuador, es considerado un tema relevante; sin embargo, no existe suficiente información por ser una problemática actual.

A su vez, se determina que el objetivo de la investigación es conocer los hábitos cognitivos, afectivos, ejecutivos y éticos, modificados durante la cuarentena por Covid-19 en personas de 24 a 60 años que residen en Ecuador.

\section{Metodología}

Se realizó un estudio cuantitativo de alcance descriptivo mediante encuestas, acerca de hábitos cognitivos, ejecutivos, afectivos y éticos. Se incluyen estudios en los que se aplicó encuestas y revisión de bibliografía (Montero \& León, 2002), estos estudios son producto de mediciones, el objetivo es recolectar información para que así sea factible el describir la situación o problema (Hernández, 2006). Se trabajó con 173 de entre 24 a 60 años que residían en Ecuador y se encontraban viviendo en estado de cuarentena por el Covid-19. 
A su vez, se realizaron las muestras homogéneas que se orientan a estudiar procesos y episodios de un grupo social con características similares. Para la adquisición de la muestra se usó el muestreo de bola de nieve, técnica en donde los individuos seleccionados reclutan a nuevos participantes entre sus conocidos (Hernández, 2006). En este sentido, se utilizó la encuesta como herramienta para poder conocer posibles modificaciones de hábitos. La encuesta contiene un conjunto de 22 preguntas semiestructuradas, se vio modificada por criterios de coherencia, adicionalmente, cuenta con tres pruebas piloto realizadas (Hernández, 2006). Las referencias de preguntas enfocadas en los hábitos se escogieron de dos cuestionarios preexistentes, inicialmente del Ayuntamiento de Alicante Concejalía de Sanidad y Consumo (2014) del programa de educación para la salud hábitos de vida saludable en familia, y en segundo lugar, la encuesta de hábitos de alimentación y sueño de González Allende (2014).

\section{Resultados}

En el primer hábito que se denomina cognitivo, se adquirieron los siguientes resultados más representativos. En las preguntas sobre modificación de hábitos cognitivos las respuestas elegidas con mayor frecuencia integran lo siguiente:

El $45,8 \%$ de las respuestas perciben que no tuvieron ningún cambio. Mientras que el $33,1 \%$ afirma haber experimentado confusión y desorientación durante la cuarentena. Seguido por dificultad en la toma de decisiones en un $25,90 \%$. En las respuestas agregadas por la muestra, el $0,60 \%$ experimentó dudas sobre comportamiento social y un $0.6 \%$ de dificultad para acostumbrarse al cambio, al tiempo de $1,2 \%$ productividad e introspección, un 1,2\% pensamiento del pasado y con $7,2 \%$ el cuestionamiento de pensamiento espiritual y religioso.

En la siguiente Figura 1 se presentan las puntuaciones porcentuales en los hábitos cognitivos durante la cuarentena con la encuesta de 4 tipos de hábitos de los 173 participantes de la muestra. Se enfoca dificultad en la toma de decisiones, confusión y desorientación, cuestionamiento de creencias y ninguna reacción mencionada, las otras respuestas fueron agregadas por las personas. 


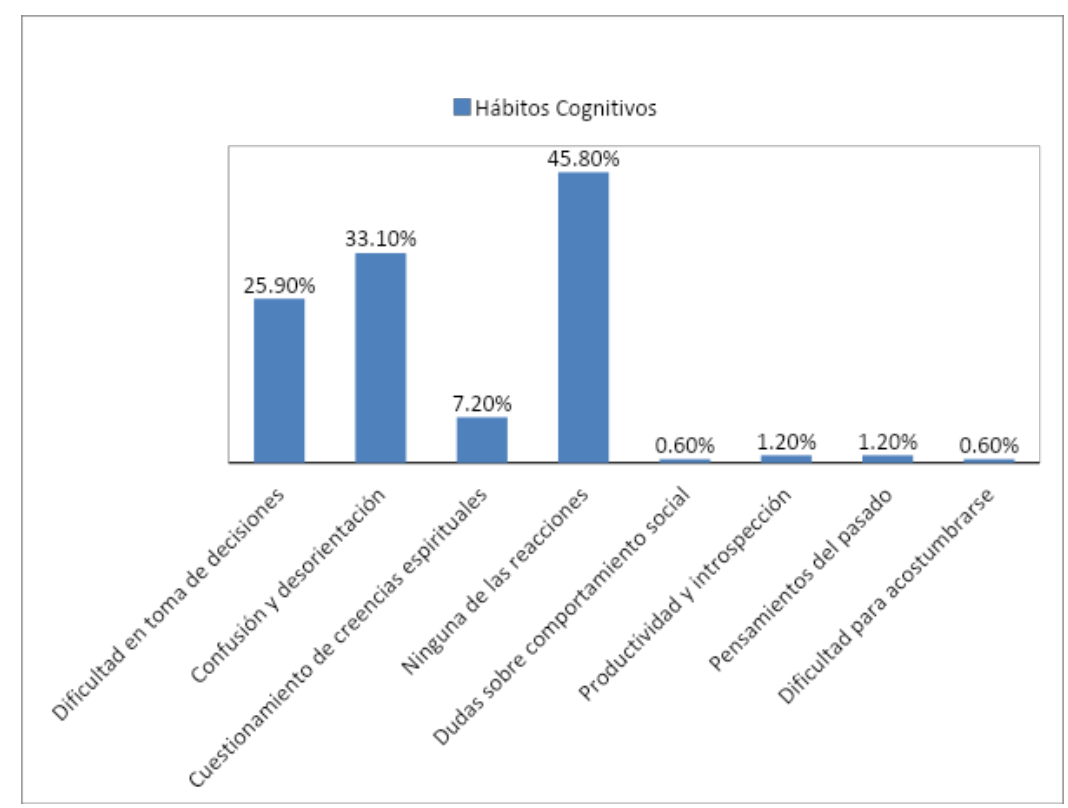

Figura 1. Puntuaciones porcentuales en los hábitos cognitivos

Fuente: elaboración propia

Además, se evidenció que el $57 \%$ de la muestra perciben cambios en los hábitos cognitivos referentes a la dificultad en toma de decisiones, desorientación y confusión de pensamientos y cuestionamiento de creencias. Mientras el $43 \%$ no percibían ningún cambio, lo que significa que la percepción de modificación de hábitos cognitivos prepondera durante la cuarentena (Figura 2).

\section{Hábitos Cognitivos: percepción de hábitos modificadas.}

¿ Si perciben modificación № perciben modificación

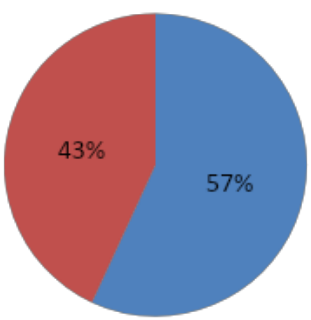

Figura 2. Puntuaciones porcentuales en los hábitos cognitivos percibidos como modificados

Fuente: elaboración propia

En otra pregunta, las respuestas seleccionadas con frecuencia son las siguientes: 
El $67,9 \%$ de las personas tienen preocupación por el desastre, seguido por un $32,7 \%$ de falta de atención en las actividades. Seguido del $25,5 \%$ de pensamientos desordenados. En respuestas menos significativas se adquirió la siguiente información:

El 3.6\% no evidenció ninguna reacción, seguido por el $14.5 \%$ que percibieron dificultad en resolver problemas. El $18.2 \%$ percibió problemas de concentración y memoria, finalmente, el $21,2 \%$ percibió preocupación por detalles insignificantes y significativos.

En la Figura 3 se evidencian las puntuaciones porcentuales en los hábitos cognitivos percibidos como modificados en el ámbito de pensamientos desordenados, falta de atención, preocupación por el desastre, problemas de concentración y memoria, preocupación por detalles insignificantes, dificultad en resolver problemas.

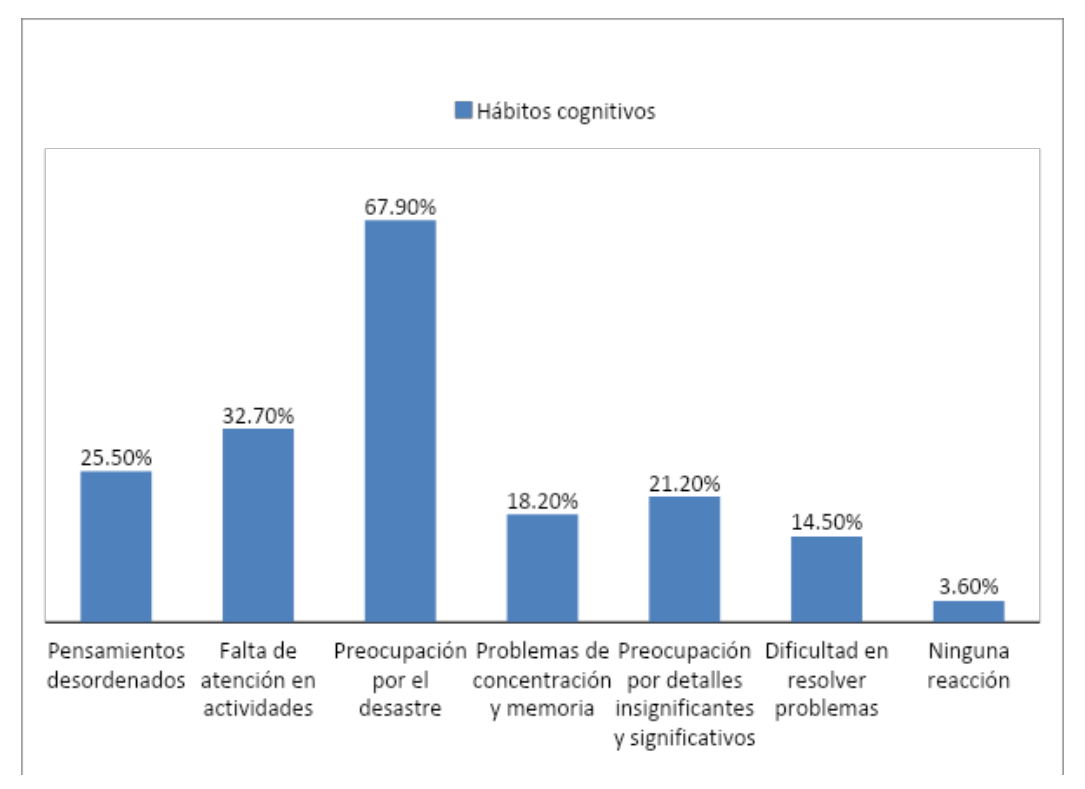

Figura 3. Pensamientos, atención, preocupación y problemas.

Fuente: elaboración propia

En una pregunta aledaña, el 59\% de personas afirman que perciben modificación en hábitos cognitivos en el ámbito de los siguientes criterios: pensamientos desordenados, falta de atención, preocupación por el desastre, problemas de concentración y memoria, preocupación por detalles insignificantes, dificultad en resolver problemas. Mientras el $41 \%$ de la muestra no percibe modificación, lo que significa que la muestra percibe que los hábitos cognitivos han sido modificados durante la cuarentena. Por otro lado, el $41 \%$ no evidencia modificación de hábitos cognitivos.

En la Figura 4 se puede ver la representación de las puntuaciones porcentuales en los hábitos cognitivos percibidos como modificados en el ámbito de pensamientos desordenados, falta de atención, preocupación por el desastre, problemas de concentración y memoria, preocupación por detalles insignificantes, dificultad en resolver problemas y otras respuestas colocadas por la muestra con la encuesta de 4 tipos de hábitos. 


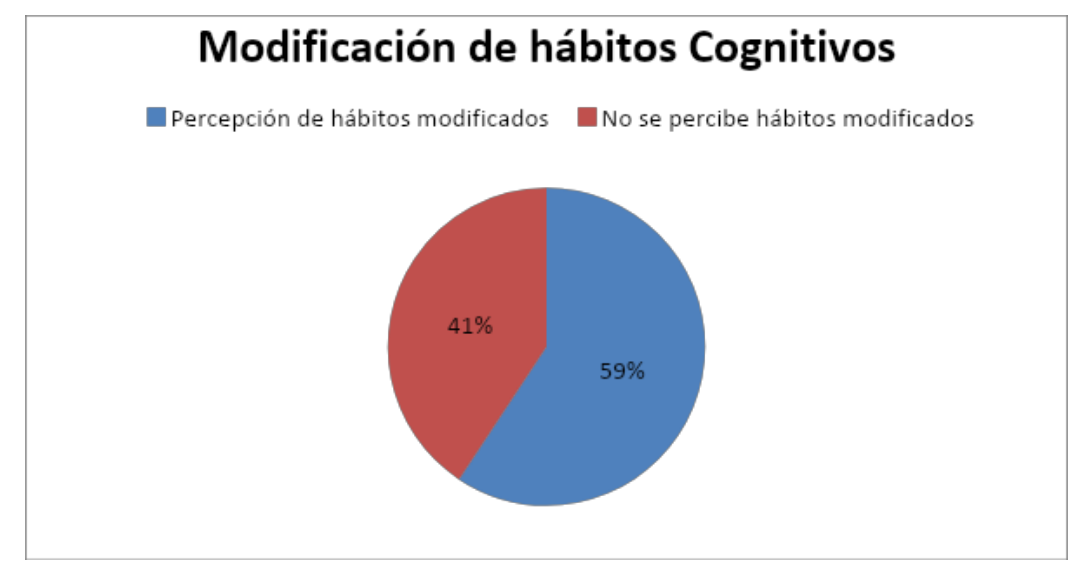

Figura 4. Percepción de hábitos modificados

Fuente: elaboración propia

De acuerdo a los hábitos afectivos, los resultados relevantes fueron los siguientes:

El $79.72 \%$ de la muestra ha tenido contacto con su familia y amigos/as. Seguido por el $14,5 \%$ de personas que se han aislado completamente. Finalmente, $12,5 \%$ de la muestra optaron por la opción de dependencia por una o más personas. En respuestas menos significativas, el $0.6 \%$ percibió independencia en sus relaciones sociales. Asimismo, el $0.6 \%$ se refirió al sentimiento de tranquilidad en la interacción social. Finalmente, el 8,9\% percibieron conflictos en la interacción social, como se ve en la Figura 5.

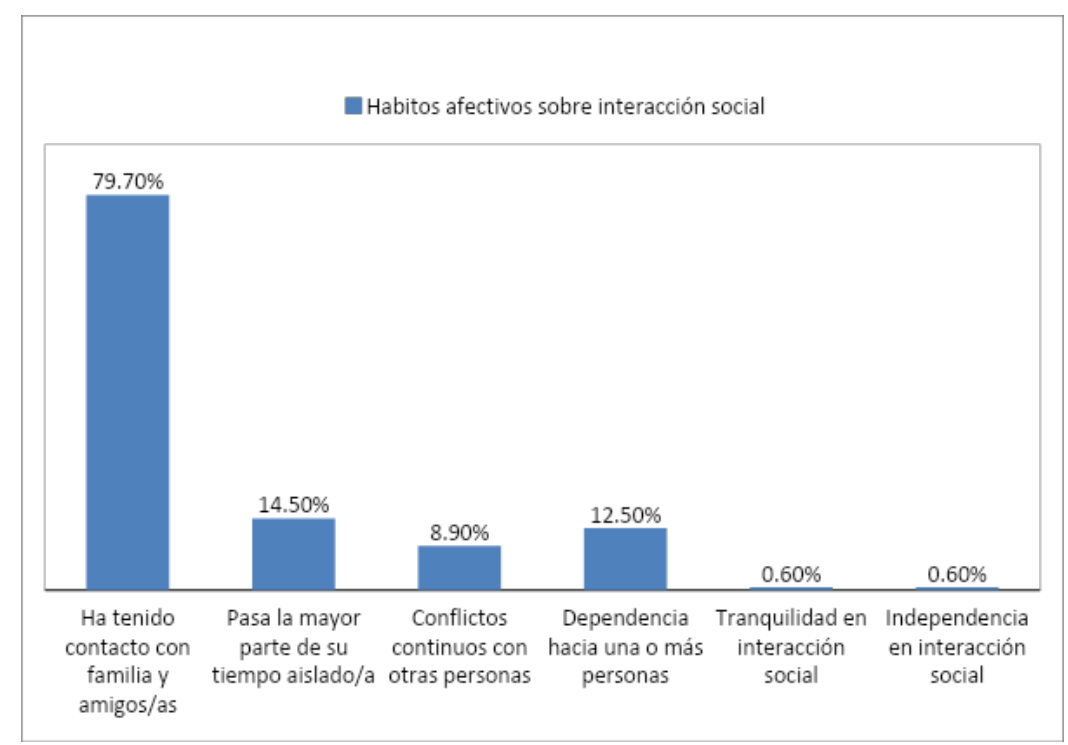

Figura 5. Hábitos afectivos sobre interacción social

Fuente: elaboración propia

Mientras que el $65 \%$ de la muestra ha preservado sus hábitos afectivos relacionados a la interacción social, siendo lo mismo antes, como durante la cuarentena. El 35\% percibe que hay modificación, tal como se evidencia en la Figura 6. 


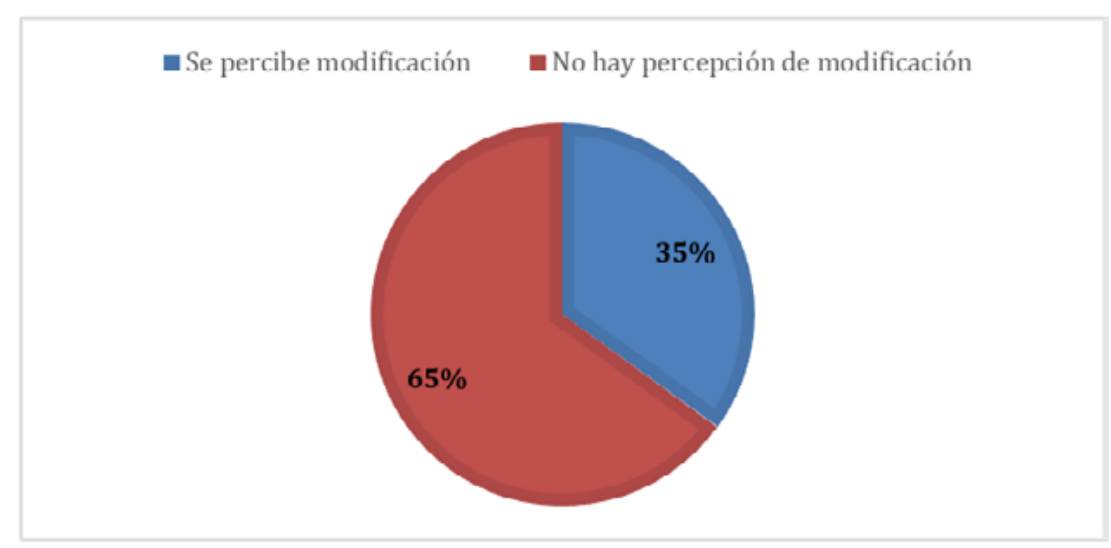

Figura 6. Percepción de modificación

Fuente: elaboración propia

Respecto a las emociones sentidas durante la cuarentena (Figura 7), las respuestas seleccionadas con frecuencia son las siguientes: el $66,3 \%$ de la muestra ha sentido tristeza, seguido por $63,2 \%$ de la muestra que seleccionó ansiedad y estrés, finalmente, el $30,8 \%$ ha sentido ira durante la cuarentena.

En respuestas menos significativas se adquirió que el 0,6\% de la muestra sintió impotencia. El $0,6 \%$ sintió pereza, seguido por el $2,4 \%$ que percibió preocupación.

El 10,1\% agregó la respuesta de haber sentido culpa. Finalmente, el 13,6\% no sintió ninguna emoción negativa durante la pandemia.

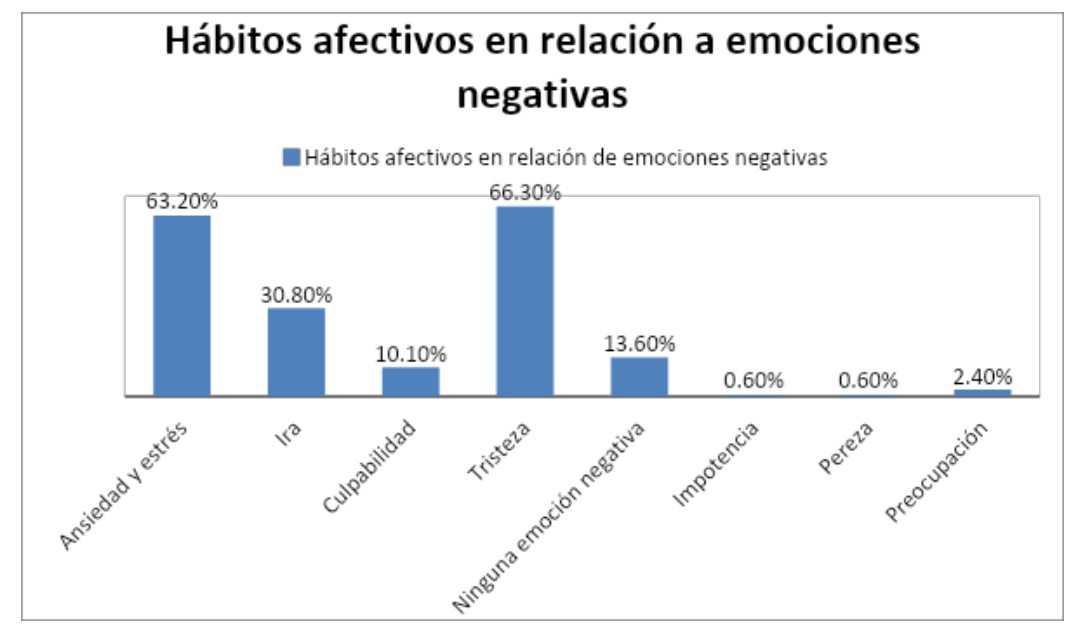

Figura 7. Emociones sentidas durante la cuarentena

Fuente: elaboración propia

En otra pregunta sobre la percepción de modificación en los hábitos afectivos (Figura 8), el 65\% de la población percibió cambios en hábitos afectivos durante la cuarentena. Mientras que el $35 \%$ no evidencia modificación. 


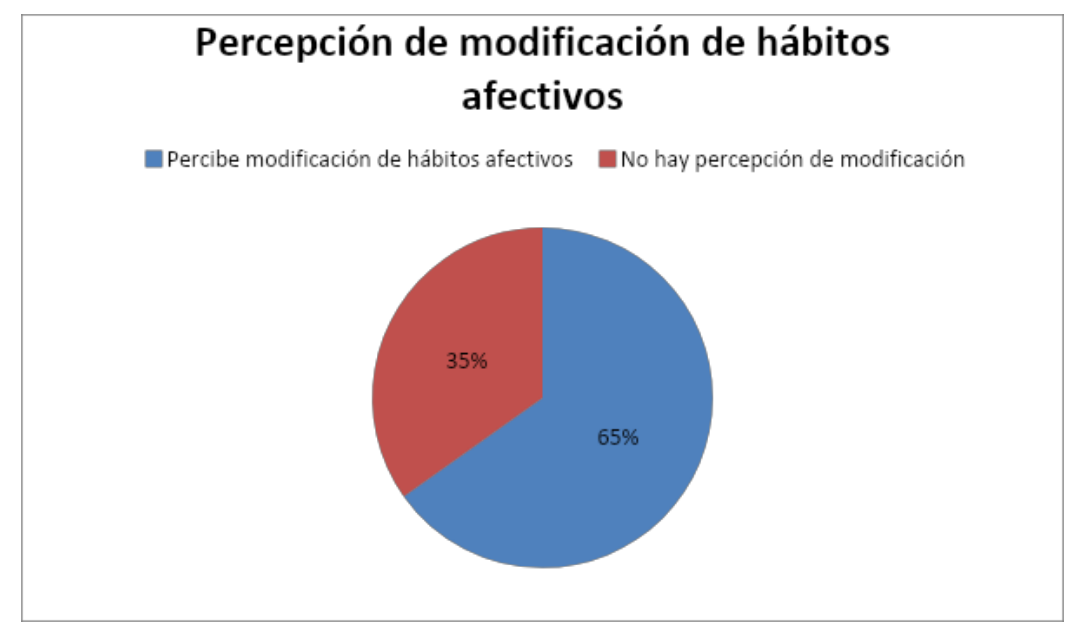

Figura 8. Percepción de modificación en los hábitos afectivos

Fuente: elaboración propia

En cuanto a cambios emocionales en cuarentena (Figura 9), las respuestas más significativas fueron que el $55,2 \%$ de la muestra sintió sensibilidad emocional, seguida por el miedo, sensación que ha sentido el $46,4 \%$. Asimismo, el $41,6 \%$ ha sentido fatiga y cansancio. Finalmente, el $29 \%$ de personas han percibido cambios de humor. Todas las reacciones muestran falta de control emocional vinculadas a la cuarentena.

En respuestas menos significativas: el 0,6\% de la muestra agregó que había sentido fuerza mental, y el $16,7 \%$ no evidenció ninguna reacción.

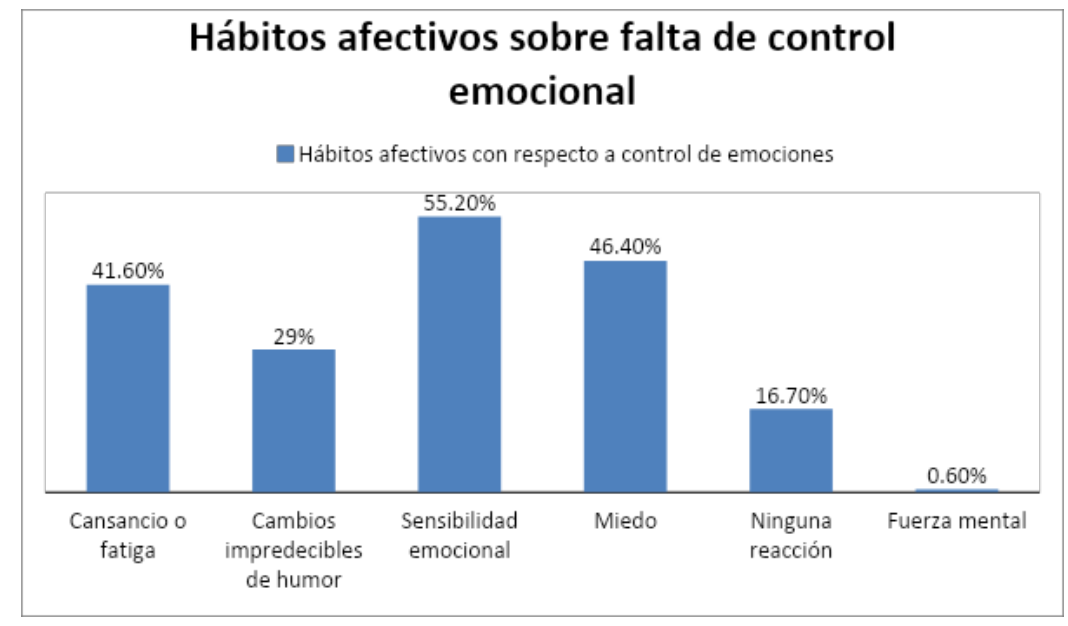

Figura 9. Cambios emocionales

Fuente: elaboración propia

Adicionalmente, el $70 \%$ demostró modificación en relación a los hábitos sociales y la falta de control emocional, y el 30\% no evidencia modificación, así como se puede ver en la Figura 10. 


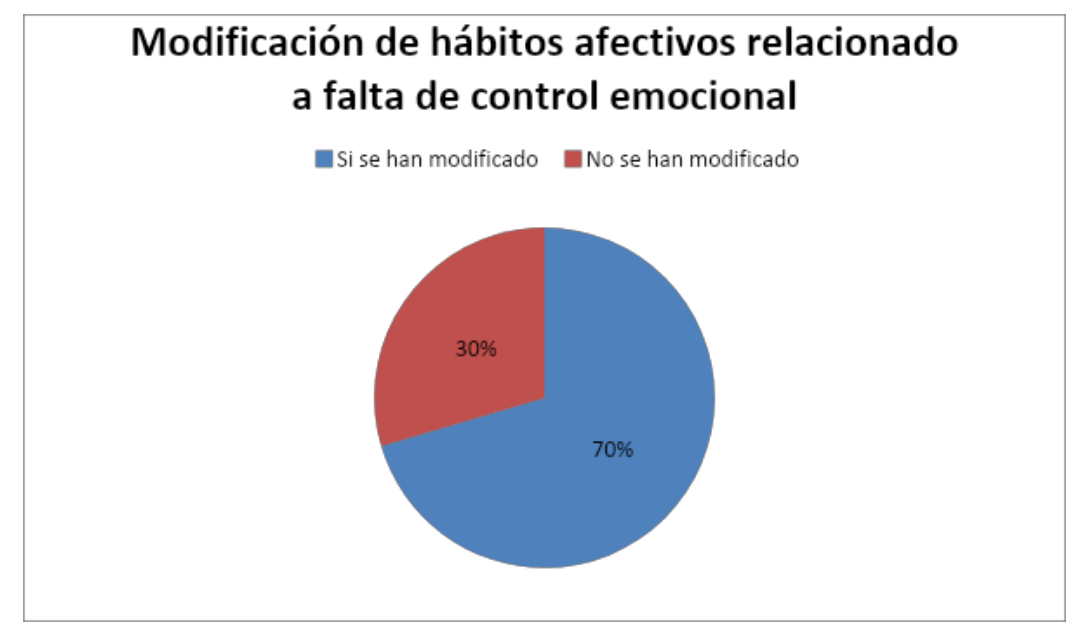

Figura 10. Modificación en relación a los hábitos sociales y la falta de control emocional

Fuente: elaboración propia

Respecto a los hábitos ejecutivos en cuanto a dificultades en dormir (Figura 11), se pudo evidenciar que el $46,8 \%$ de la muestra poseen problemas de insomnio y se les dificulta conciliar el sueño, seguido del $34,3 \%$ de personas que se despiertan a distintas horas en la noche. Otra respuesta fue que el $27,8 \%$ de las personas respondieron que no tienen problemas para dormir.

En respuestas menos significativas, la muestra agregó que el $0,6 \%$ poseen pesadillas, el $0.6 \%$ poseen necesidad de tomar siestas, el $0,6 \%$ poseen pesadillas, el $0,6 \%$ duermen de 5 a 6 horas y no sienten que han descansado y el $1,2 \%$ duerme 5 a 6 horas sintiendo que han descansado. 


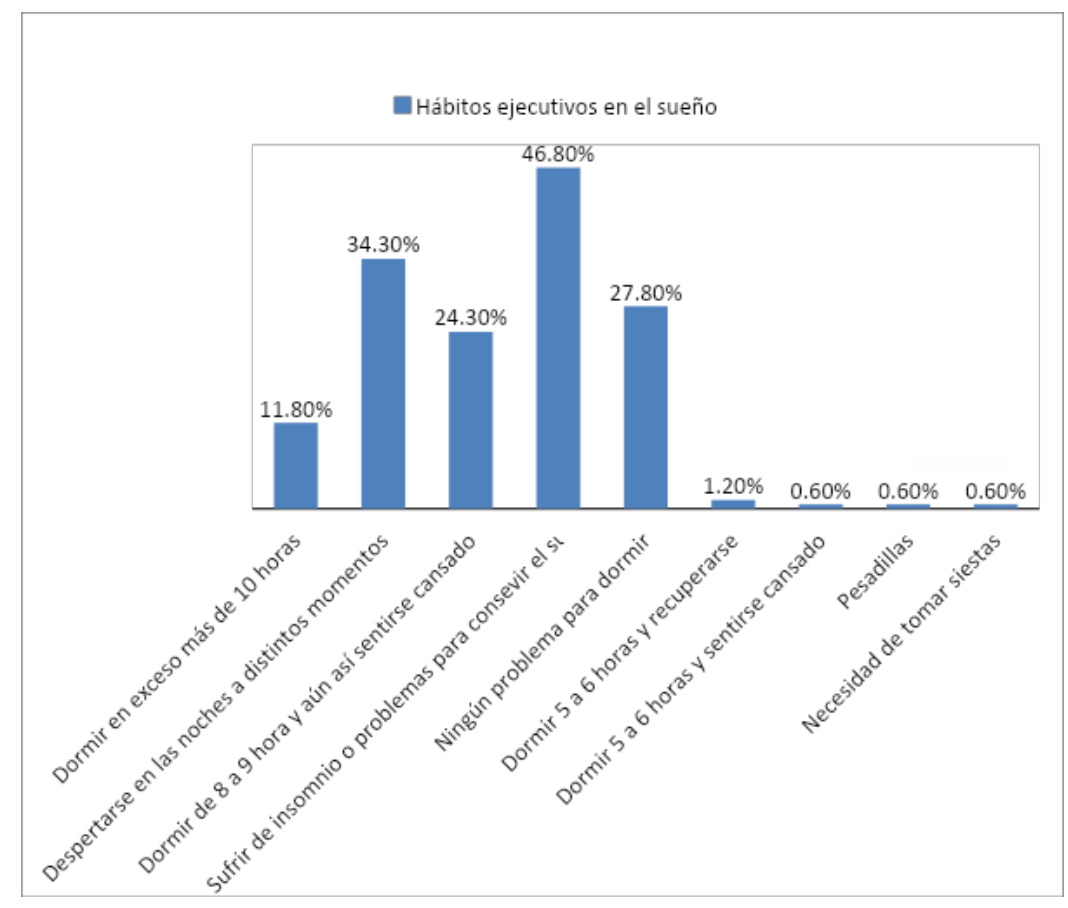

Figura 11. Hábitos ejecutivos en cuanto a dificultades en dormir

Fuente: elaboración propia

Sobre la percepción de los hábitos del sueño (Figura 12), el 73\% de la muestra considera que existe una modificación durante la cuarentena, mientras que el $27 \%$ consideran que no hay modificación. Prevalece la modificación en hábitos de sueño.

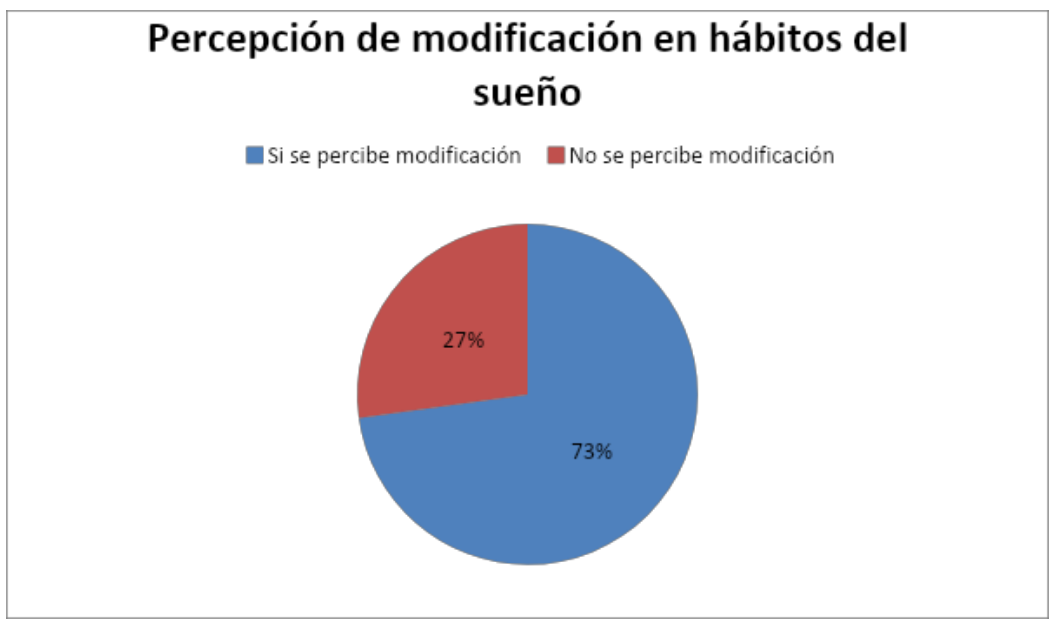

Figura 12. Hábitos del sueño

Fuente: elaboración propia

En otra pregunta relacionada con los hábitos ejecutivos centrados en actividades físicas (Figura 13 ), encontramos que el $56 \%$ de la muestra realiza actividades físicas durante la cuarentena, mientras que el $44 \%$ no realizan actividades físicas. 


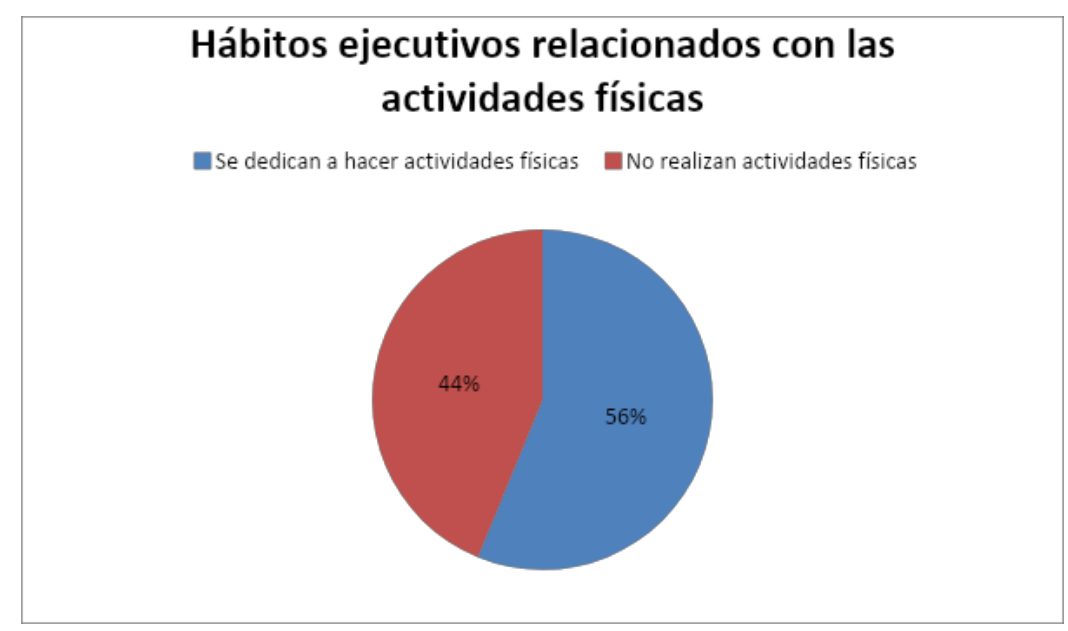

Figura 13. Hábitos ejecutivos centrados en actividades físicas

Fuente: elaboración propia

En la pregunta sobre autocontrol (Figura 14), el 78\% de la muestra percibe que ha sido reflexivo durante cuarentena, regulando respuestas agresivas e impulsivas, seguido por el $16 \%$ de personas que han percibido impulsividad.

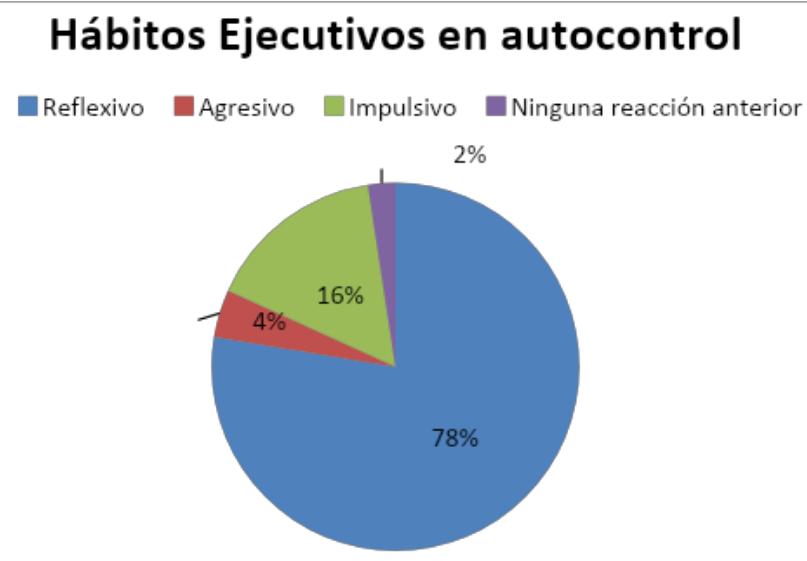

Figura 14. Autocontrol

Fuente: elaboración propia basado en "encuesta sobre 4 hábitos" por R. Navas.

En la pregunta de percepción de modificación de hábitos, el 57,1\% de la muestra percibe que este hábito cognitivo de autocontrol no ha cambiado, y el $43 \%$ no percibe modificación, como se ve en la Figura 15. 


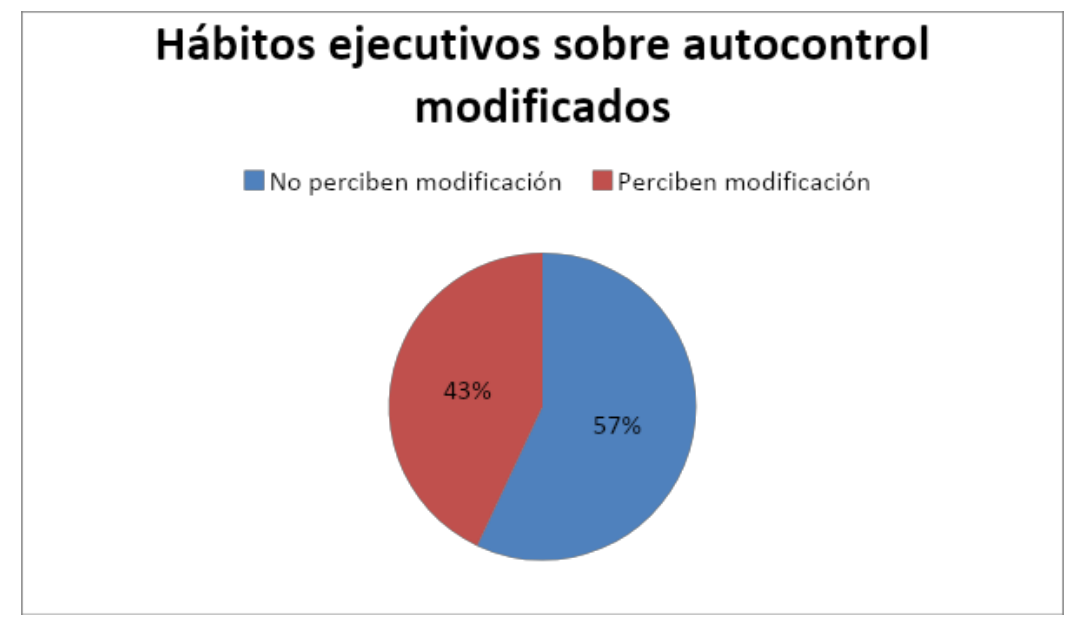

Figura 15. percepción de modificación de hábitos

Fuente: elaboración propia

Según la percepción de modificación en horarios en hábitos alimenticios (Figura 16), el 58\% no percibe modificación, mientras que el $42 \%$ percibe modificación.

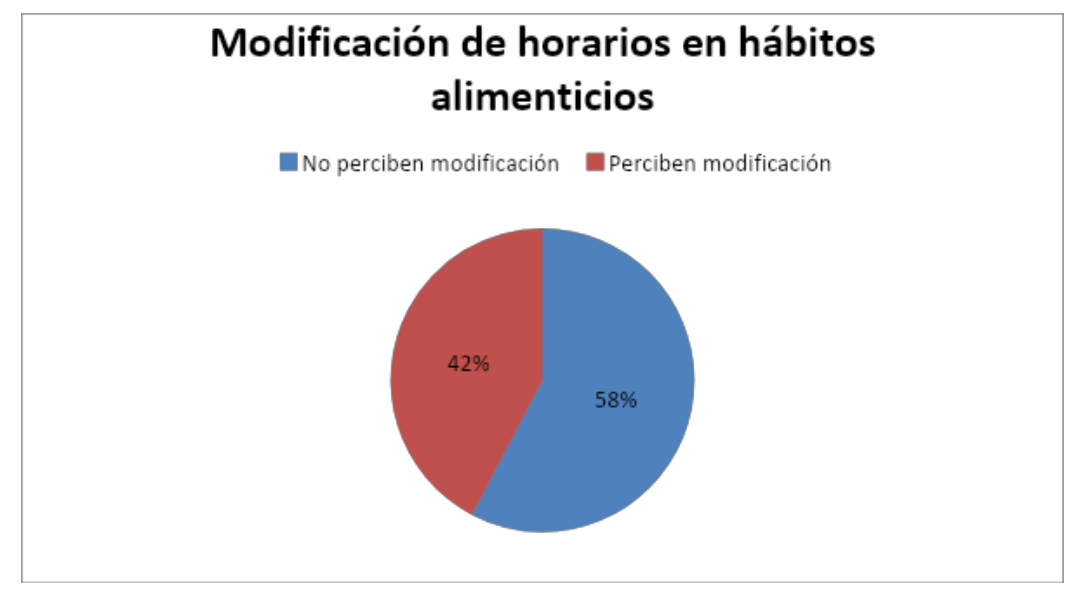

Figura 16. Modificación en horarios en hábitos alimenticios

Fuente: elaboración propia

Con respecto a la modificación en la organización del cronograma de actividades (Figura 17), el $64 \%$ de la muestra percibe una modificación, mientras que el $36 \%$ no percibe modificación alguna. 


\section{Percepción de modificación de hábitos ejecutivos ocn relación a la modificación de horarios}

Eercepción de modificación $\quad$ No perciben modificación

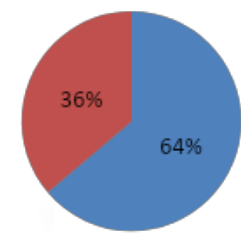

Figura 17. Modificación en la organización del cronograma de actividades

Fuente: elaboración propia

En la percepción de hábitos de estudios modificados, el $63 \%$ de la muestra percibe modificación en hábitos de estudio durante la cuarentena y el $36 \%$ no percibe modificación alguna (Figura 18).

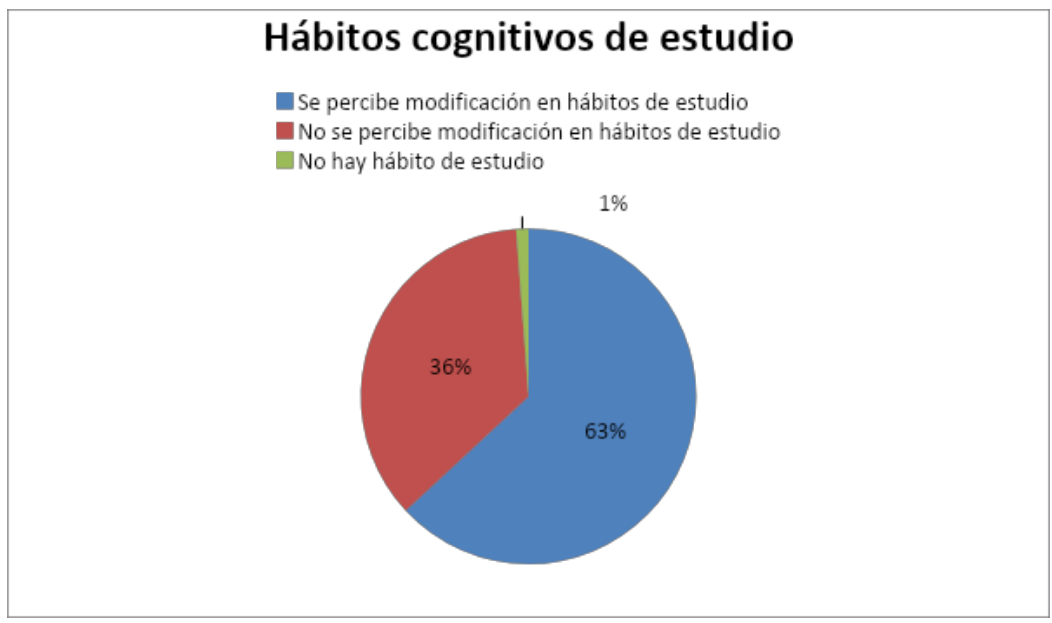

Figura 18. Percepción de hábitos de estudios modificados

Fuente: elaboración propia

El cuarto hábito es el ético (Figura 19), aquí se adquirieron los siguientes resultados relevantes. Dentro de las respuestas seleccionadas con frecuencia, el $92 \%$ de la muestra no percibe modificación en cuestionamiento de creencias religiosas y espirituales, mientras el $8 \%$ percibe modificación de hábitos. Lo que significa que no se evidencia modificación significativa. 


\section{Modificación en hábitos éticos: creencias espirituales y religiosas}

Modificadas No modificadas

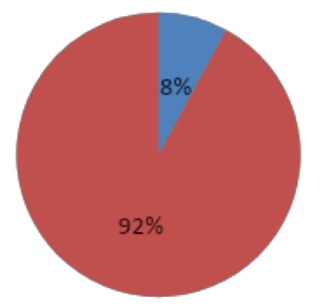

Figura 19. Modificación en el hábito ético

Fuente: elaboración propia

En otra pregunta, la muestra seleccionó las siguientes conductas solidarias que se representan en la Figura 20: el $86.7 \%$ afirmó quedarse en casa y salir solamente para compras, se concientiza con lo que se está atravesando.

En segundo lugar, el $71.1 \% \%$ de las personas colaboran en casa tanto con higiene y cocina.

Finalmente, el $17.5 \%$ de la muestra han ayudado económicamente o donaciones en instituciones y a personas vulnerables.

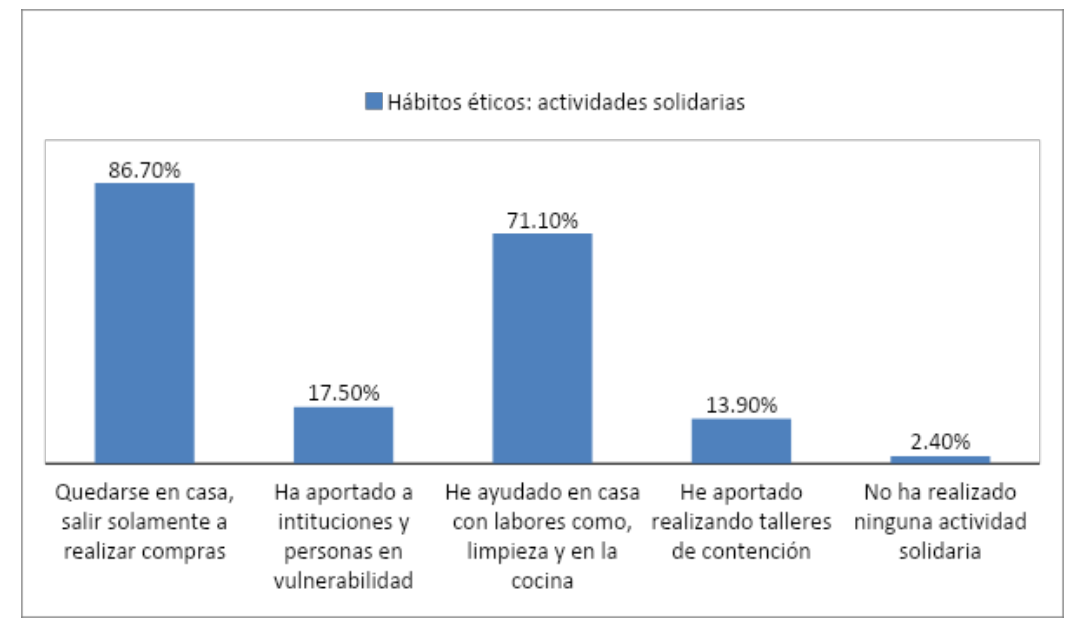

Figura 20. Conductas solidarias

Fuente: elaboración propia

Dentro de hábitos éticos (Figura 21) las respuestas más frecuentes sobre valores fueron las siguientes: el $76.7 \%$ de las personas ha aplicado la paciencia, seguido por el respeto en los/as demás con un porcentaje de $75.5 \%$, además, se presentó un $62.6 \%$ de solidaridad. Finalmente, el valor de sinceridad fue seleccionado por $46.5 \%$ de la muestra. 


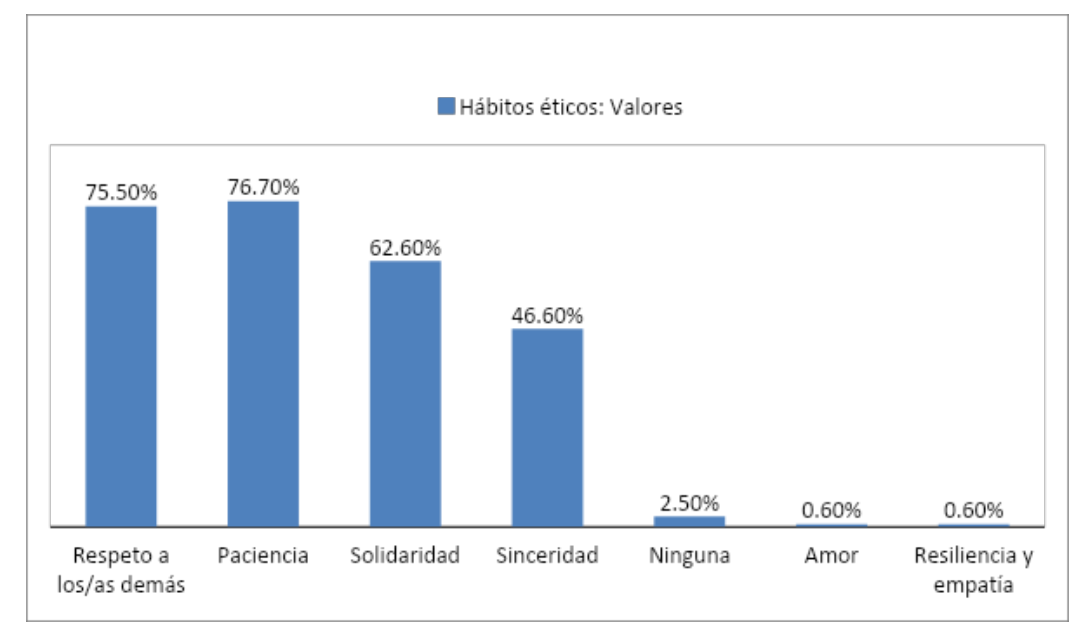

Figura 21. Hábitos éticos

Fuente: elaboración propia

Adicionalmente, el $86 \%$ de la muestra integrando una respuesta que se encontraba en otras opciones, se evidencia que las personas seguían los mismos valores antes y durante la cuarentena. Mientras el 14\% percibe cambios en hábitos éticos, como se evidencia en la Figura 22.

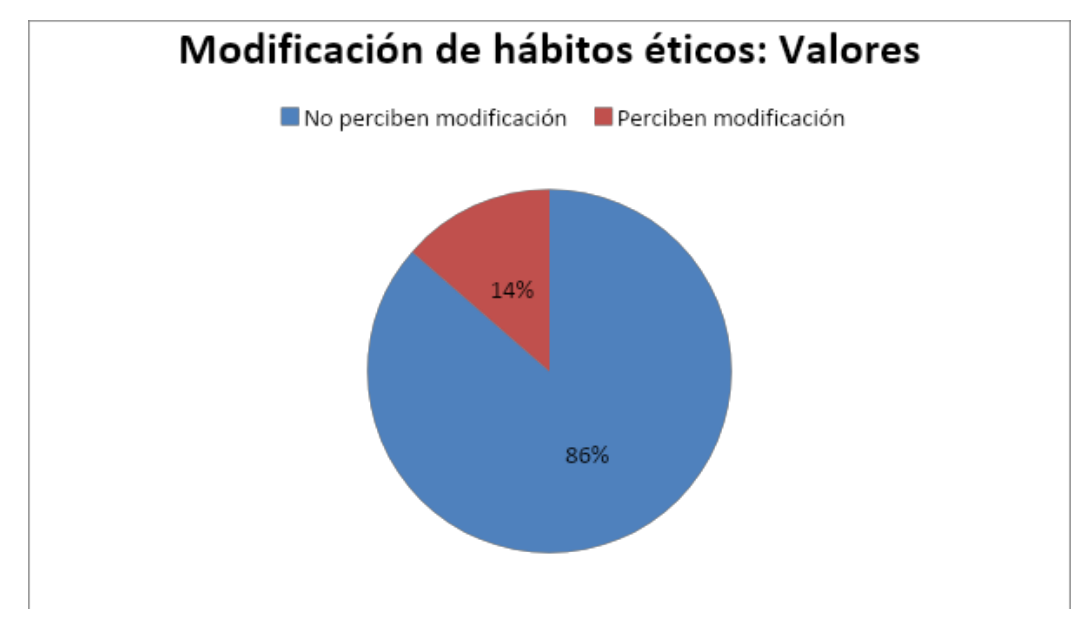

Figura 22. Cambios en los hábitos éticos

Fuente: elaboración propia

En la investigación, en cuanto a la percepción de hábitos cognitivos modificados tales como, la toma de decisiones, el autocontrol y en las creencias espirituales, se evidenció que el $54 \%$ de la muestra no perciben cambios durante la cuarentena. En este caso, las variables que son parte del hábito cognitivo han perdurado, lo que se vincula con lo indicado por Marina (2012); Vélez (2008) los cuales afirman que los hábitos cognitivos permiten generar nuevas opciones y destrezas, por lo que en una situación de crisis se pueden mantener habilidades que nos permitan continuar dilucidando lo que es razonable y lo que no. Esta afirmación no concuerda con la teoría de crisis, 
puesto que cuando existen sucesos precipitados, los 4 tipos de hábitos deberían modificarse (Estrada et al., 2017).

En cuanto a otro tipo de modificación de hábitos cognitivos, las personas pudieron percibir preocupación por el desastre y falta de atención con mayor frecuencia. El 59\% de la muestra percibió cambios en los hábitos cognitivos referente a la capacidad de atención y percepción. En esta misma línea, Estrada et al. (2017) consideraron que a partir de las crisis existen varios tipos de modificaciones en varias funciones cognitivas.

Con respecto a los hábitos afectivos, el 79,7\% de la muestra ha tenido contacto con su familia y amigos, en la siguiente pregunta, el $65 \%$ de la muestra no percibe modificación en los hábitos afectivos respecto a la interacción social. Lo que sigue la línea de Marina (2012), quien sostiene que los hábitos son conductas que se repiten y aprenden a tal punto que pueden mantenerse.

Por otra parte, en los hábitos afectivos, lo que la muestra sintió con más frecuencia fue tristeza, ansiedad y estrés. De hecho, el $65 \%$ de las personas perciben modificación en los hábitos afectivos durante la cuarentena, ello significa que las emociones negativas son experimentadas con mayor frecuencia. Otras emociones seleccionadas con frecuencia fueron: sensibilidad emocional y miedo. El $70 \%$ de las personas afirman percibir cambios emocionales y falta de autocontrol emocional. Lo que concuerda con Trujillo (2018), quien indicó que durante la crisis española del 2008 en España se evidenciaron cambios a nivel de hábitos afectivos. Desde esta perspectiva, otros autores como Estrada et al. (2014) y Solano et al. (2017) se contraponen a los resultados, afirmando que las reacciones durante una crisis se ven modificadas y pueden volver a equilibrarse luego de un tiempo, si se optó por una intervención adecuada o la personalidad de las personas es de tipo resiliente.

De acuerdo a los hábitos ejecutivos, la mayor parte de personas perciben problemas de insomnio y problemas de conciliar el sueño. Además, algunos se despiertan durante la noche a distintas horas. El $73 \%$ de la muestra afirmó que sus hábitos de sueño se modificaron, de acuerdo a los autores anteriormente mencionados, a partir de la crisis existen varias modificaciones a nivel ejecutivo como las alteraciones del sueño.

En cuanto al hábito del autocontrol conductual, el $78 \%$ de personas posee conductas reflexivas. De acuerdo a la persistencia de la conducta, el $57 \%$ de la muestra no percibe ningún cambio en su autocontrol conductual, como indicó Marina (2012), Trujillo (2018) y Vélez (2008), hay hábitos que perduran durante el tiempo porque han sido aprendidos mediante repetición y son necesarios para que el ser humano continúe reconociendo qué es racional y qué no lo es.

En lo que se refiere a los hábitos de alimentación, el $58 \%$ de personas no perciben modificación en horarios de alimentación. Asimismo, en el estudio de Cabrera (2006), los horarios de alimentación se encuentran intactos, lo cual es una reacción inesperada para la teoría de la modificación en estados de crisis (Estrada et al., 2017).

Respecto a los horarios para realización de actividades, el 53\% realiza actividades siguiendo un orden flexible. Las respuestas sobre la percepción de horarios modificados evidenciaron que el 
$64 \%$ de la muestra no percibe un cambio durante la cuarentena. Se mantiene la organización en los horarios de actividades. Según Cabrera (2006), Marina (2012) y Vélez (2008) el afianzamiento de hábitos adecuados en horarios de actividades puede mantenerse.

En los hábitos de estudio, el $63 \%$ percibe modificación en hábitos de estudios, seguido por el $36 \%$ que no percibe modificación, y el 1\% expresa que no posee hábitos de estudio, en este sentido, se asocia con la teoría de Estrada et al. (2017), a partir de la crisis existen varios tipos de modificaciones en los hábitos de estudio.

En cuanto a hábitos éticos, no se evidenció modificación significativa en cuestionamiento en creencias religiosas y espirituales. Respecto a valores, el $76,7 \%$ de las personas ha aplicado la paciencia. Seguido por el respeto en los/as demás con un porcentaje de $75.5 \%$, seguido por un porcentaje de $62,6 \%$ de solidaridad. En la siguiente pregunta, el $86 \%$ de la muestra han conservado los mismos valores antes y durante la cuarentena. La muestra no percibe modificación en hábitos éticos. En este sentido, en el artículo de Marina (2012) los hábitos éticos están constituidos por varias virtudes y valores que tienen como objetivo su acción y su potencialización, eso podemos visualizar en las preguntas dónde los hábitos persisten.

\section{Conclusiones}

Durante la cuarentena, cuándo analizamos los hábitos cognitivos, se evidenció lo siguiente, con relación a la toma de decisiones, el autocontrol y en las creencias espirituales, no existe percepción de modificaciones, aun así, las personas perciben ciertos cambios durante la cuarentena.

En cuanto a lo relacionado con percibir preocupación por el desastre y falta de atención, las personas de la muestra percibieron una modificación. Mientras que, en los hábitos afectivos, se concluye que las personas mantienen contacto con familia y amigos/as, por lo que no se visualiza modificación. Con respecto a emociones negativas si hay una modificación percibida durante la cuarentena, las personas sintieron tristeza, ansiedad, sensibilidad al cambio y miedo, esto denota falta de autocontrol emocional; no obstante, las reacciones evidenciadas se encuentran dentro de los parámetros cuándo existen situaciones de crisis o cambios abruptos.

Respecto a los hábitos ejecutivos, tenemos las siguientes conclusiones: no se evidencia cambio en actividades físicas significativas, otro de los factores que no se modificaron fue lo que respecta al autocontrol conductual, dónde la muestra no percibió un cambio, esta mantiene sus hábitos de alimentación y continúan siguiendo los mismos horarios que antes de la cuarentena, en hábitos de estudio, las personas perciben que se han modificado sus horarios durante la cuarentena.

Finalmente, los hábitos éticos se mantienen, la muestra no percibe cambios antes y durante la cuarentena. Continúan desarrollando y aplicando valores tales como la paciencia, el respeto, la solidaridad. 


\section{Referencias}

Allende, G. (2014). Encuesta de hábitos de alimentación y sueño. https://studylib.es/doc/5434375/encuesta-de-h\%C3\%A1bitos-de-alimentaci\%C3\%B3n-y-sue\%C3\%B10

Ayuntamiento de Alicante Concejalía de Sanidad y Consumo. (2014). Programa de educación para la salud. Hábitos de vida saludable en familia. Encuesta. https://umhsaludable.umh.es/files/2014/06/Programa-de-educaci\%C3\%B3n-para-la-salud.-H\%C3\%A1bitos-de-vida-saludable-en-familia.pdf

Ayuntamiento de Vitoria Gasteiz. (2020). Retos de la solidaridad. https://www.vitoria-gasteiz.org/docs/wb021/ contenidosEstaticos/adjuntos/es/78/31/7831.pdf

Caballero García, P. A., \& Campos Riaño, J. D. (2018). Hábitos saludables y componentes psicológicos que afectan a los cuidadores informales. [Tesis de grado, Universidad Santo Tomas]. Repositorio institucional Universidad Santo Tomas http://hdl.handle.net/11634/16416

Cabrera, E. (2006). Educación, factor determinante para la modificación de hábitos alimenticios. [Tesis de grado, Universidad de las Américas de Puebla]. Repositorio Institucional UDLAP. http://repositorio.udlap.mx/ xmlui/handle/123456789/12735

Echeburúa, E., \& Corral, P. D. (2007). Intervención en crisis en víctimas de sucesos traumáticos: ¿Cuándo, cómo y para qué? Psicología conductual, 15(3), 373-387. https://www.behavioralpsycho.com/producto/ intervencion-en-crisis-en-victimas-de-sucesos-traumaticos-cuando-como-y-para-que/

EL COMERCIO. (16 de abril de 2020). Ecuador confirma 8225 enfermos con coronavirus; 1035 personas han muerto en el contexto del covid-19. https://www.elcomercio.com/actualidad/ecuador-covid19-muestras-fallecidos-contagiados.html

Estrada, A., Gutiérrez, L., \& Hernández, I. (2017). Manual básico de primeros auxilios. Universidad De Guadalajara, Centro Universitario de Ciencias de la Salud. https://www.cucs.udg.mx/sites/default/files/adjuntos/ manual primeros auxilios 2017.pdf

Hernández, R. (2006). Metodología de Investigación. Mc Graw Hill.

Leija, G., Aguilera, V., Lara, E., Rodríguez, J., Trejo, J. \& López, M. (2011). Diferencias en la modificación de hábitos, pensamientos y actitudes relacionados con la obesidad entre dos distintos tratamientos en mujeres adultas. Revista Latinoamericana de Medicina Conductual, 1(2), 19-28. https://www.redalyc.org/ pdf/2830/283021986003.pdf

MacMillan, N. (2007). Valoración de hábitos de alimentación, actividad física y condición nutricional en estudiantes de la Pontificia Universidad Católica de Valparaíso. Revista chilena de nutrición, 34(4), 330-336. http:// dx.doi.org/10.4067/S0717-75182007000400006 
Marina, J.A. (14 de diciembre de 2012). Los hábitos, clave del aprendizaje. joseantoniomarina.net. https://www. joseantoniomarina.net/articulos-en-prensa/los-habitos-clave-del-aprendizaje/

Montero, I., \& León, O. (2002). Clasificación y descripción de las metodologías de investigación en Psicología. International Journal of Clinical and Health Psychology, 2(3), 503-508. https://www.redalyc.org/ pdf/337/33720308.pdf

Organización Mundial de la Salud. (2020). Enfermedad Coronavirus 2019 (COVID-19), Reporte de situación, 67. https://www.who.int/docs/default-source/coronaviruse/situation-reports/20200327-sitrep-67-covid-19. pdf?sfvrsn=b65f68eb 4

RTVE. (2020). Mapa del coronavirus en el mundo: casos, muertes y los últimos datos de su evolución. https:// www.rtve.es/noticias/20200421/mapa-mundial-del-coronavirus/1998143.shtml

Solano-Pinto, N., Canales, I. S., Cézar, R. F., López, S. C., \& Bardera, C. P. (2017). Hábitos saludables en la primera infancia y en sus familias. Una invitación a la reflexión. DEMETRA: Alimentação, Nutrição \& Saúde, 12(4), 803-821.

Trujillo, L. (2018). Cambios en los hábitos saludables de los españoles por la crisis de 2008. [Tesis de pregrado, Universidad de la Laguna].

Vélez, A. (2008). La adquisición de hábitos como finalidad de la educación superior Educación y Educadores. Educación y educadores,11(1), 167-180. https://educacionyeducadores.unisabana.edu.co/index.php/eye/ article/view/723/1701 
Copyright (c) 2021 Rosa Estefanía Navas Espinosa

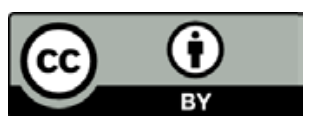

Este texto está protegido bajo una licencia internacional Creative Commons 4.0.

Usted es libre para Compartir-copiar y redistribuir el material en cualquier medio o formato - y Adaptar el documento - remezclar, transformar y crear a partir del material-para cualquier propósito, incluso para fines comerciales, siempre que cumpla las condiciones de Atribución. Usted debe dar crédito a la obra original de manera adecuada, proporcionar un enlace a la licencia, e indicar si se han realizado cambios. Puede hacerlo en cualquier forma razonable, pero no de forma tal que sugiera que tiene el apoyo del licenciante o lo recibe por el uso que hace de la obra.

Resumen de licencia - Texto completo de la licencia 